\title{
sist \\ Perspectivismo na filosofia da ciência: um estudo de caso na física quântica
}

\author{
Décio Krause \& Jonas Rafael Begker Arenhart<smiles>C#CC(C)CC</smiles>

RESUMO

Neste artigo, apresentaremos uma visão particular do desenvolvimento de teorias científicas que denominamos (inspirados em Ortega y Gasset) "perspectivismo”. Discutiremos como, através desse enfoque, é possível compatibilizar diversas descrições aparentemente distintas e incompatíveis de uma suposta realidade que se investiga. Fazemos isso distinguindo entre a "realidade" $(R)$ e a "descrição empírica da realidade" (Re).Aceitando que podemos ter diversas descrições empíricas de uma mesma realidade, discutimos o caso particular em que esse esquema é utilizado nos debates atuais acerca da ontologia da física quântica, em especial da mecânica quântica não relativista. Como se sabe, essa teoria é compatível com distintas ontologias (ou metafísicas), em particular, pode ser vista como comprometida com uma ontologia de indivíduos, mas também com uma ontologia de não indivíduos. Ambas as alternativas são plausíveis e merecem ser desenvolvidas. Propomos que o perspectivismo aqui apresentado é neutro com relação à escolha de uma metafísica (ontologia), bem como aos debates entre realistas e antirrealistas em filosofia da ciência. Concluímos que passar do pluralismo aceito pelo perspectivista ao realismo ou antirrealismo envolve comprometimento com teses mais robustas acerca da relação entre realidade e descrição da realidade.

Palavras-Chave • Perspectivismo. Mecânica quântica. Realismo. Antirrealismo. Ontologia quântica. Metafísica.

\begin{abstract}
Dentro de la humanidad cada raza, dentro de cada raza cada individuo es un órgano de percepción distinto de todos los demás y como un tentáculo que llega a trozos de universo para los otros inasequibles. La realidad, pues, se ofrece en perspectivas individuales (Ortega y Gasset, 1998, p. 52).
\end{abstract}

\section{INTRODUÇÃO}

A investigação científica é, sem dúvida, uma das formas mais bem sucedidas de interrogação do mundo. Dificilmente algum tipo de investigação da natureza pode comparar-se ao seu sucesso. Diante desse fato, parece razoável que também a ontologia, en- 
tendida como a disciplina filosófica responsável pelo estudo daquilo que há em seus aspectos mais gerais, poderia beneficiar-se, quando estivesse de algum modo aliada à ciência. De fato, alguns filósofos chegam a sugerir que as disputas filosóficas medievais, em particular na ontologia, podem e devem ser abolidas: bastaria tomarmos o testemunho da ciência para descobrirmos o que há no mundo, juntamente com suas características mais gerais.

Essa sugestão, por sua vez, levanta diversas dificuldades que os filósofos foram rápidos em identificar. Para começar, não fica claro o que estamos chamando de teoria nesse caso. De modo geral, o que os cientistas chamam de teoria não está de modo algum bem delimitado, e uma compreensão precisa do significado desse termo requer investigação filosófica. Com efeito, é reconhecido que aquilo que muitas vezes os cientistas tratam como sendo, para todos os efeitos, uma mesma teoria pode ser formulada de diferentes modos, como ocorre, por exemplo, com a mecânica clássica, que pode ser formulada seguindo-se o modo original de Newton, ou seguindo-se Lagrange, ou ainda ao estilo proposto por Hamilton, dentre outros. Apesar das diferentes formulações de uma "mesma teoria" poderem ter as mesmas aplicações em determinados domínios, cada uma delas pode ser vista como priorizando certos aspectos de descrição de uma realidade, em detrimento de outros. Assim, como vamos escolher qual delas é melhor com respeito às entidades que devemos tomar como existindo? Há uma maneira simples de decidir essa questão?

A dificuldade anterior pode ser expandida em pelo menos duas direções. Primeiramente, podemos imaginar uma situação em que diferentes teorias são capazes de dar conta de um mesmo domínio da realidade. Nesse caso, temos uma situação que é conhecida como a subdeterminação das teorias pelos dados, ou primeira tese da subdeterminação. Situações, nas quais ocorrem subdeterminações desse tipo, são consideradas como criando um grande problema para aqueles que desejam tirar conclusões acerca da ontologia mais adequada partindo das teorias científicas atualmente dadas. De fato, se temos mais de uma teoria igualmente plausível e retirando o mesmo tipo de apoio dos dados disponíveis, então deveremos apelar para virtudes não empíricas em nossa escolha entre essas teorias, as chamadas "virtudes teóricas", como simplicidade, beleza e poder explicativo. Isso, no entanto, é visto por muitos filósofos como ilegítimo, dado que não há razões para acreditar que uma teoria mais simples, por exemplo, seja uma melhor candidata a estar mais próxima da verdade do que uma teoria mais complicada (cf. Devitt, 2002).

A segunda direção na qual podemos expandir a dificuldade criada acima diz respeito à questão acerca de uma teoria ser considerada mais fundamental do que outra. Em tal caso, um mesmo domínio pode ser estudado por diferentes teorias que o inves- 
tigam em diferentes escalas. Um mesmo objeto, por exemplo, um cão, pode ser estudado por biólogos, por químicos, por físicos etc. sob diferentes perspectivas, cada uma levando em conta determinados aspectos do animal. Em geral, os físicos de partículas afirmarão estar estudando o "cão realmente", pois nada existe de "mais fundamental" que as partículas básicas estudadas na física de partículas. $\mathrm{O}$ problema para aqueles que estiverem interessados em estudar ontologia em conjunção com a ciência é evidente nesta situação. Devemos considerar que o cão, como usualmente entendido, é "real"? Ou apenas suas moléculas seriam reais, apenas os átomos que o compõem? Ou ainda, não seria real apenas uma determinada configuração em forma de cão composta de partículas elementares?

Todas essas dificuldades certamente estão formuladas de modo bastante impreciso, e muitas outras poderiam ser apresentadas. São questões fundamentais acerca da relação entre ontologia e ciência, e não é fácil sequer estabelecer bases claras nas quais as diferentes respostas possam ser descritas e comparadas. Nosso interesse aqui é formular uma abordagem ao problema que permita que nos beneficiemos dos pluralismos apresentados pela ciência (calcados em diferentes perspectivas) e tornemos mais claras as noções vagas que mencionamos acima. Propomos uma forma de perspectivismo em filosofia da ciência, tendo em vista, em particular, estudos de fundamentos da ciência e de ontologia. Começaremos apresentando um esquema acerca da elaboração de teorias científicas na seção 1. Na seção 2, discutiremos como esse esquema pode ser útil com relação aos estudos de fundamentos. Nesse particular, vamos considerar alguns dos problemas de ontologia em mecânica quântica. Essas discussões seguem na seção 3, onde, em vista da completude do artigo, apresentamos em linhas gerais o problema acerca da ontologia da teoria quântica. Na seção 4, aplicaremos as ferramentas conceituais do perspectivismo a esse caso em particular, argumentando que podemos escalrecer certas disputas existentes. Por fim, na seção 5 , consideraremos brevemente casos em que diferentes descrições da realidade, operando em escalas de grandeza diferentes, podem ser entendidas como compatíveis entre si sem que seja necessário adotar a tese mais forte, segundo a qual a única teoria que, de fato, trata com o real seria aquela que se ocupa das entidades em menor escala no momento (tomada como mais fundamental em sentido ontológico). Nesse sentido, evitamos assumir de partida qualquer compromisso com formas de reducionismo radicais, que sugerem que se eliminem de uma ontologia aquelas entidades que não pertencem aos níveis mais fundamentais. 


\section{UMA ABORDAGEM DAS TEORIAS GIENTÍFICAS}

Suponha-se que objetivemos investigar certa porção de uma suposta realidade. Um biólogo pode estar interessado em uma determinada população de peixes, um físico, nos buracos negros de uma galáxia, um economista, em uma determinada parcela da população consumidora, e assim por diante. Se formos realistas (e há realistas de diversas tendências), aceitaremos que existe uma "realidade" independente de nossas vontades ou mentes. O mundo estaria aí (talvez um pouco diferente), mesmo se não existíssemos. Para os chamados realistas científicos, nossas teorias (pelo menos as melhores) contam a verdade acerca desse mundo, pelo menos parcialmente, e não seria por milagre que elas dão certo: as teorias de fato funcionam nas aplicações práticas e nas suas predições, por descreverem corretamente como o mundo é em traços pelo menos aproximados (este é, em linhas gerais, o que de denomina de "argumento do não milagre”, uma das principais armas dos realistas científicos). Sem discutir em detalhes a questão, vamos assumir simplesmente que, para a investigação científica, faz sentido assumir que há algo além de nós mesmos que desejamos investigar. Pelo menos no tocante às teorias físicas, parece razoável aceitar o que nos diz Auyang quando afirma que "as teorias físicas são sobre coisas (...), [pois] pretendem descrever situações concretas e referir-se a objetos particulares. Soluções testáveis experimentalmente garantem sozinhas o significado físico das teorias" (Auyang, 1995, p. 152).

Vamos seguir o físico e filósofo francês d'Espagnat e chamar esse "algo" que desejamos investigar no mundo físico de "realidade $R$ ” e, como ele, assumir (algo kantianamente) que $R$ permanece, para nós, "velada" ou inacessível (cf. d’Espagnat, 2003, 2006), ainda que a possamos conhecer cientificamente (mais sobre isso adiante). Tudo o que dispomos (supostamente) a respeito de $R$ são dados fenomênicos, informações coligidas de nossa interação fenomênica com o domínio (via observação, experimentos com aparelhos, o que quer que seja), que ele denomina de "realidade empírica Re". Nossas teorias forneceriam apenas uma descrição "alegórica" do mundo físico através das descrições da realidade empírica. Nesse sentido, um realista aceitaria que a ciência, pelo menos em suas teorias mais maduras e bem estabelecidas, nos dá descrições verdadeiras da realidade, de modo que ela não fica para sempre velada, enquanto que um construtivista ou kantiano aceitaria fazer e manter a distinção entre realidade $R$ e realidade empírica $R e$.

Deve ficar claro que nossa investigação de $R$ não é feita de simples observações (em sentido amplo). Se não estivermos preparados, podemos ficar horas observando os traços em uma câmara de bolhas sem concluir nada do que um físico pode facilmente concluir, ou observar por horas uma população de peixes sem perceber nada do que um biólogo treinado pode perceber. Dito diretamente, nossas observações, nossas 
análises dos dados fenomênicos, nossos modelos de dados, para empregar um termo devido a Patrick Suppes, dependem de nosso conhecimento prévio, das teorias e concepções que carregamos em nossa bagagem (inclusive do ponto de vista cultural). Essa observação foi feita por Einstein ao jovem Heisenberg, e é lembrada por este último em várias passagens, o que mostra a importância que ele lhe atribuiu. Segundo Heisenberg, Einstein lhe teria ensinado que são as teorias que nos permitem dizer o que pode ser observado, e que "somente a teoria, ou seja, o conhecimento das leis naturais, permite que, partindo de nossas impressões sensoriais, possamos deduzir os fenômenos subjacentes". Einstein teria dito ainda que "é heuristicamente útil ter em mente o que de fato se observou. Mas, em princípio, é um grande erro tentar fundamentar uma teoria apenas em grandezas observáveis. Na realidade, dá-se o inverso. É a teoria que decide o que podemos observar" (Heisenberg, 1996, p. 77-9). Com isso, desejamos enfatizar que nossa análise de $R$, via $R e$, já utiliza conhecimentos científicos e mesmo culturais, contidos em nossa bagagem prévia (infelizmente, algumas vezes entram em cena preconceitos ideológicos, que a ciência deveria em princípio evitar). Se desejamos teorizar a respeito de $R$, o fazemos com os dados que dispomos em Re, com base em nosso conhecimento prévio (nossas teorias), e levando em conta $R e$.

Muitas vezes, fazemos uso de dispositivos heurísticos $H$ como modelos de aeroplanos, de uma molécula de DNA, uma maquete de uma hidroelétrica ou, para simular (ou "modelar") as moléculas de um gás, algo como uma mesa de bolas de bilhar. A partir de insights provenientes desses esquemas, conhecimentos anteriores, experiências etc. elaboramos (via de regra, fazendo uso de alguma matemática) o que vamos denominar de "modelo matemático $\mathrm{Mm}$ ", ou teoria informal - no sentido de que ela não está, via de regra, axiomatizada -, visando dar conta de Re e, indiretamente, de $R$. É claro que há várias possibilidades de elaboração de Mm. Cada cientista tem sua visão do contexto, os seus conhecimentos prévios, modos peculiares de entender uma situação. Vamos fixar-nos em um desses Mm. O modo de construir ou elaborar tal teoria tem sido muito discutido na literatura, e não nos interessa aprofundar essa questão aqui. Basta que reconheçamos que a atividade científica é, em grande medida, uma atividade conceitual. O biólogo mencionado acima utiliza noções (conceitos) como as de "espécie", "ambiente", "PH da água" etc.; o físico que estuda buracos negros valese dos conceitos da relatividade geral, enquanto que o economista utiliza noções como as de "mercado" e "demanda", dentre outras. Esses conceitos podem ser imaginados (pelo menos idealmente) coligidos, ou agrupados, em uma estrutura, uma estrutura matemática no caso das teorias físicas, mas que pode adquirir outras formas nas demais disciplinas, como a economia ou a psicanálise (que faz uso de conceitos como "inconsciente", "ego", "rejeição”, dentre outros). Como estamos interessados na física, vamos restringir-nos a falar dessa disciplina. Assim, podemos pensar em um Mm, 
tal como caracterizado por uma estrutura conjuntista, já que para as teorias físicas usuais toda a parafernália matemática que precisamos pode ser obtida em uma teoria de conjuntos como ZFG (a teoria Zermelo-Fraenkel com o axioma da escolha). Aqui aparece um dado importante, pois, na física, elaboramos nosso $\mathrm{Mm}$ fazendo uso de uma matemática (e, portanto, de uma lógica), apesar de quase sempre estarmos utilizando outras teorias (físicas) e concepções, que nos auxiliam a "modelar" nossa Re.

Muitas vezes, para produzir um $M m$, o cientista não dispõe dos conceitos adequados, necessitando criá-los, o que faz, quase sempre, valendo-se de analogias entre teorias ou de conhecimentos anteriores. Assim, a noção de "partícula" muda conforme a teoria física que estejamos empregando, mas o mesmo termo continua a ser utilizado, apesar de adquirir significados distintos (cf. Falkenburg, 200\%, cap. 6). Da mesma forma, muitas vezes o cientista não dispõe de uma matemática adequada, necessitando criá-la. Newton, por exemplo, criou os cálculos diferencial e integral para dar conta de seus anseios em física, e Fourier desenvolveu o que se chama hoje "análise de Fourier" a partir do estudo da transmissão do calor. Esses são fatos históricos. O que importa é que há uma matemática disponível com a qual o cientista opera para elaborar seu $\mathrm{Mm}$. Esse modelo, ou teoria informal, está carregado de significado, ou seja, seus termos e conceitos acham-se interpretados nos moldes da Re, e indiretamente, da visão que tem o cientista de $R$. Assim, quando o biólogo fala da reprodução de seus peixes, está mesmo falando da reprodução dos animais que constituem sua parcela da realidade sob investigação, e não de entidades supostamente abstratas, independentes de qualquer interpretação.

No entanto, esse vínculo com $R$ deve ser visto com cautela. Quando dizemos, por exemplo, que o sistema solar é um modelo da teoria mecânica de Newton, estamos fazendo uma idealização. O sistema solar verdadeiro (seja lá o que queiramos dizer com isso), estritamente falando, não obedece às leis de Kepler-Newton. Por exemplo, as órbitas dos planetas não são elipses perfeitas, assim como não há corpo que não esteja sujeito à ação de forças etc. O que temos como modelo, e que representa o sistema solar em nossa visão é, como dito, uma abstração, uma idealização.

Os modelos matemáticos, $M m$, que usualmente chamamos de "teorias", via de regra são estabelecidas informalmente, isto é, sem o recurso do método axiomático, como ilustram a teoria da evolução darwiniana, ou a física de Galileu. Em outras oportunidades, esses modelos matemáticos, como preferimos denominá-los, podem ser desenvolvidos axiomaticamente, ou semi-axiomaticamente, como o comprova o exemplo da mecânica newtoniana). É essencialmente nesse nível informal que trabalha o cientista. As duas teorias da relatividade foram apresentadas desse modo, bem como as primeiras formulações da mecânica quântica (pré-von Neumann), feitas por Heisenberg (a 'mecânica de matrizes') e Schrödinger (mecânica ondulatória), a teoria 
da evolução de Darwin e muitas outras. Ou seja, o cientista propriamente dito geralmente pára por aí, desenvolvendo teorias informais $M m$ e estudando os modos como essas teorias o auxiliam a explicar a realidade empírica, e indiretamente, a realidade propriamente dita.

Cabe observar que o "modelo" da dupla hélice do DNA, de Watson e Crick, ou os planos inclinados de Galileu mostram que a palavra "modelo" entra de modo essencial no discurso filosófico, mas o faz em diversas acepções que devem ser distinguidas com cautela pelo filósofo. O dispositivo heurístico que, por vezes, o cientista usa simplesmente o auxilia a entender a teoria (ou a elaborá-la, pois pode permitir a simulação de situações que se supõem ocorrem na realidade) e, portanto, a entender seus verdadeiros modelos, que são constructos matemáticos. Convém insistir que os modelos das teorias científicas são constructos matemáticos, e não a "realidade" propriamente dita. Retornando, se, como dissemos, para o cientista, a elaboração de um modelo matemático (ou teoria informal) pode bastar, isso em geral não contenta o fundacionista, que deseja mais, em especial, conhecer a estrutura das teorias elaboradas. Podemos dizer que ao fundacionista interessa um estudo metateórico. Entra então em cena uma terceira etapa, a da elaboração de uma teoria $T$ em sentido estrito, no sentido em que tencionamos aqui empregar o termo "teoria”, ou seja, uma versão axiomatizada, ou mesmo formalizada, da teoria informal. Claro que, assim como uma mesma coleção de dados fenomênicos pode dar origem a diferentes teorias informais, ou modelos matemáticos, uma mesma teoria informal pode dar origem a diferentes axiomatizações ou formalizações, logo, a diferentes "teorias". Exatamente por ser uma teoria informal, seus contornos não são bem delimitados, de forma que as versões axiomáticas ou formais de um mesmo Mm podem inclusive ser incompatíveis entre si. Como este último ponto nos interessa sobremaneira, deixaremos para falar dele mais abaixo. Uma teoria axiomatizada ou formal, contrariamente à teoria informal, ganha um caráter de abstração, no sentido de não pressupor necessariamente uma interpretação particular de seus conceitos primitivos, ainda que possa ter um "modelo pretendido". Isso faz que ela possa ter vários modelos, no sentido de estruturas matemáticas que satisfaçam seus postulados, dependendo da interpretação particular que se adote. Assim, uma determinada teoria $T$ pode ter vários modelos abstratos $M a$, inclusive não isomorfos, pois a teoria pode não ser categórica. (Note-se que a palavra "modelo" está sendo usada em diferentes acepções, como é comum na ciência).

Uma questão interessante parece-nos ser a seguinte. Hoje em dia, talvez a passagem de um Mm para uma teoria $T$, no sentido acima, seja um ideal inalcançável em certas áreas da física. Um exemplo típico é o da busca por uma teoria $T$ da gravitação quântica, que uniria as teorias quânticas de campos e a relatividade geral. Da mesma forma, o próprio modelo padrão da física de partículas carece de uma teoria $T$ 
unificadora. A física de hoje funciona como um conjunto de dispositivos (equações, pseudoteorias informais) heurísticos que o cientista aplica caso a caso, sem que haja uma unificação satisfatória em termos de postulados, conceitos primitivos e outras coisas que caracterizariam o que tradicionalmente chamaríamos de "teoria", algo tendo princípios unificadores e métodos únicos. Talvez se possa dizer que, hoje, pelo menos em certas áreas, a axiomatização de uma disciplina é um mero ideal, constatação que, no entanto, somente o tempo pode ajudar a estabelecer.

\section{A RELEVÂNGIA Dos ESTUdos FUNDAGIONISTAS}

Qual é a importância do trabalho fundacionista? Podemos destacar várias questões que são postas em relevo pela análise dos fundamentos de uma teoria (informal) científica, tais como:

(1) Delineamento preciso de sua contraparte matemática, incluindo sua lógica subjacente. Isso implica, por exemplo, em conhecer-se, tanto quanto possível - e de modo claro na versão formal da teoria - sua linguagem e aparato dedutivo. Recordemos que em um constructo teórico objetiva-se, dentre outras coisas, a elaboração de argumentos, sejam eles dedutivos ou indutivos. Uma inferência (centremo-nos nas inferências dedutivas) depende de uma lógica, que a valida. Assim, dependendo da lógica que se utiliza, certos tipos de inferência feitos por alguns (por exemplo por alguém que professa a lógica clássica) podem não ser aceitos por outros (por exemplo, por alguém que advogue uma forma de intuicionismo a La Brouwer). (2) Explicitação de seus conceitos básicos (dependendo da axiomática particular adotada). Ou seja, delineamento do que é assumido como primitivo e do que pode ser definido a partir disso, de que princípios adotar etc. (3) Possibilidade de generalização, no sentido de perceber outros universos (ou domínios) aos quais a teoria (inicialmente informal e carregada de significado) possa ser aplicada. Com efeito, uma vez axiomatizada ou mesmo formalizada (em especial neste último caso), a teoria torna-se algo abstrato, desvinculada do estudo dos objetos iniciais que motivaram seu desenvolvimento, podendo comportar diferentes interpretações, outras além daquela que a motivou. Popper diria que ela ganhou autonomia, tornandose um objeto daquilo que ele chama de "mundo 3" (cf. Popper, 1978).

(4) Estudo metateórico da teoria, por exemplo, buscando-se saber se valem alguns metateoremas importantes, como consistência e categoricidade 
ou, quando não houver categoricidade, se é possível provar algum teorema de representação para os modelos da teoria (cf. Suppes, 2002). Dito por alto, uma teoria $T$ admite, via de regra, uma infinidade de modelos (estruturas que satisfazem seus axiomas). Um teorema de representação busca determinar uma classe de modelos de tal forma que qualquer modelo da teoria tenha nessa classe um modelo que lhe seja isomorfo. Assim, conhecendo-se essa classe, "conhecemos" todos os modelos da teoria.

(5) Ainda que não intencionalmente, uma formulação axiomática ou formal de uma teoria pode deixar transparecer outras características da teoria informal não percebidas antes. Pelo menos na matemática, reconhece-se que isso é bastante comum. A discussão milenar sobre o postulado das paralelas na geometria euclidiana possibilitou a elaboração das geometrias não euclidianas; a axiomatização da teoria de conjuntos (iniciada com Zermelo) possibilitou que se percebesse que pode haver várias teorias de conjuntos não equivalentes, e que a noção de "conjunto" não é absoluta (a mesma em todas as formulações); a discussão da independência da hipótese do contínuo e do axioma da escolha em teorias como ZermeloFraenkel levou à constatação da possibilidade da elaboração de "matemáticas não cantorianas", para empregar a expressão de Paul Cohen. Outros exemplos podem ser buscados na literatura. Patrick Suppes (1983) discute o papel heurístico do método axiomático em Suppes.

De especial relevância para nosso trabalho aqui são os pontos (1) e (4). Conhecer a lógica subjacente e, com isso, realizar estudos metateóricos são dois aspectos muito proximamente relacionados entre si. Por exemplo, se desconfiamos que uma teoria informal apresenta inconsistências, será desejável saber se essas inconsistências são apenas aparentes, logo elimináveis, ou se fazem parte da teoria em um nível essencial. Ambas as possibilidades podem e devem ser estudadas, mas no segundo caso a lógica subjacente terá de ser tolerante com a presença de contradições, em especial, a presença de uma contradição não deverá acarretar que todas as sentenças de sua linguagem sejam deriváveis como teoremas, o que ocorreria em contextos nos quais a lógica subjacente é a clássica. Como se sabe, na lógica clássica, ex falso sequitur quodlibet (de uma contradição tudo se segue). Além disso, é interessante investigar como a lógica subjacente pode influenciar aspectos próprios, característicos, da teoria. ${ }^{\mathbf{1}}$

1 Para uma investigação nesse sentido acerca das posições de Bohr em mecânica quântica, cf. da Costa \& Krause, 2006; para um tratamento mais geral do nosso esquema perspectivista envolvendo inconsistências em física, cf. da Costa \& Krause, no prelo. 
Esses tipos de estudo já vêm sendo realizados há um bom tempo com relação às teorias matemáticas. Muito se tem descoberto acerca de matemáticas cuja lógica subjacente admite contradições, ou, para o caso mais conhecido, sobre lógicas e matemáticas alternativas de vários tipos (cf. Mortensen, 2008; Bridges, 2012). Algumas delas se prestam ao desenvolvimento de toda a matemática necessária para grande parte da física atual, outras não. Como essas matemáticas alternativas relacionam-se com a física é ainda uma questão em aberto para muitos sistemas não clássicos de lógica. De especial interesse seria o estudo de algumas matemáticas não cantorianas, as quais, via de regra, são erigidas em teorias de conjuntos nos quais o axioma da escolha não é válido de modo geral. Por exemplo, uma dessas matemáticas, a chamada matemática de Solovay, permite que se desenvolvam espaços de Hilbert nos quais todos os operadores são limitados. Como é sabido, a mecânica quântica ortodoxa, em sua formulação usual, faz uso de espaços de Hilbert em que operadores ilimitados desempenham papel central. Seria possível desenvolver uma versão da mecânica quântica em matemáticas como a de Solovay? Essas são questões relevantes para o interessado em estudos sobre os fundamentos da ciência.

Nesse mesmo campo de investigações, temos as questões relacionadas com a ontologia de uma teoria. Através de nossas teorias informais, podemos conhecer muitas características dos objetos que compõem o domínio da realidade que se estuda. Em particular, quando nossa única forma de contato com esses objetos é dada bastante indiretamente, por meio de experimentos que, em geral, interferem de modo incontrolável com os objetos medidos, como no caso da mecânica quântica, há um amplo espaço para especulação acerca da natureza metafísica dessas entidades e para diversas possibilidades de teorias metafísicas. Nesse sentido, acreditamos, há um papel central a ser desempenhado pela lógica subjacente. É ela que terá a tarefa de "codificar" algumas das principais características dos objetos tratados. Esse interesse refletiu-se no intenso estudo de lógicas alternativas associados com a mecânica quântica, desde as lógicas trivalentes propostas por Reichenbach a sistemas relacionados que buscavam captar a noção bohriana de complementaridade (cf. da Costa \& Krause, 2006), até a atual multiplicidade de sistemas de lógica quântica.

Em particular, ainda no campo das considerações acerca de sistemas alternativos de lógica para a mecânica quântica, gostaríamos de concentrar a atenção nos sistemas de lógica não reflexiva. Tais lógicas buscam captar uma intuição fundamental acerca das entidades quânticas. Segundo uma interpretação em voga desde o fundamento da "nova teoria" quântica, por volta de 1925, os objetos quânticos não são indivíduos, no sentido de não possuírem condições de identidade bem determinadas. Ou seja, não há nada na teoria que possa ser dito servir como um fundamento para afirmações de que certos objetos, em determinadas situações como quando estão emaranhados, são iguais 
ou diferentes uns dos outros (em particular, que são diferentes, pois não apresentam qualquer diferença). Schrödinger, um dos fundadores da teoria, foi enfático ao sustentar que, em muitas circunstâncias, a identidade ou diferença simplesmente não faz sentido para esses objetos (cf. French \& Krause, 2006, cap. 3).

Evidentemente enquanto estamos ainda tratando com a teoria informal, esse tipo de afirmativa permanece um tanto vaga. A identidade é um assunto a ser tratado através de estudos da lógica subjacente e da explicitação de conceitos primitivos (uma investigação acerca dos fundamentos, conforme a entendemos aqui). Nesse sentido, podemos tornar precisa a afirmação de que as entidades quânticas não possuem identidade e não são indivíduos através de um adequado ajuste na lógica subjacente. Por outro lado, para aqueles que não estão convencidos dessa tese, está aberto o caminho para que sustentem que a identidade faz completo sentido para as entidades quânticas, e assim, pelo menos dois tipos de caminho estão disponíveis no momento da formalização, cada um correspondendo, em linhas gerais, a diferentes convicções acerca de traços metafísicos apresentados pela mecânica quântica. Assim, diferentes perspectivas são possíveis de serem levadas em conta.

O que se constata é que uma teoria, assim concebida, pode ser compatível com várias ontologias, ou várias metafísicas (cf. French, 1998, 2011; French \& Krause, 2006). Vamos ver com mais detalhes do que se trata, considerando o caso específico da mecânica quântica (cf. French, 2011).

\section{As DIFERENTES METAFÍSICAS NA FÍSICA QUÂNTICA}

$\mathrm{O}$ que vimos anteriormente de forma resumida anteriormente permite-nos inferir a seguinte tese. $\mathrm{O}$ formalismo matemático da mecânica quântica não relativista, e talvez mesmo os das teorias quânticas de campos, permite associar diversas, mas pelo menos duas metafísicas (ou ontologias) distintas e de certa forma incompatíveis entre si, uma metafísica de não indivíduos e uma metafísica de indivíduos. Essa tese foi denominada de tese da subdeterminação da metafísica pela física, ou ainda, segunda tese da subdeterminação (cf. French \& Krause, 2006, p. 189 ss.), querendo dizer que a física (no caso, a teoria quântica) não determina nem impõe uma visão metafísica determinada, sendo de fato compatível com mais de uma possibilidade (perspectiva) de entendimento da natureza metafísica dos objetos quânticos. Para manter o artigo autocontido, vamos rever em linhas gerais o argumento que nos conduz à subdeterminação da metafísica pela física.

No centro da problemática que dá origem ao dilema da subdeterminação da metafísica está a alegada indistinguibilidade das partículas quânticas e o papel do chamado 
"postulado da indistinguibilidade". Para começarmos com a noção de indistinguibilidade, é interessante observar que, no que diz respeito às propriedades intrínsecas, ou seja, independentes do estado, como massa e carga elétrica, todas as partículas de um mesmo tipo, como os elétrons, são indiscerníveis, ou seja, partículas de um mesmo tipo possuem todas as propriedades independentes do estado em comum. O que podemos dizer das propriedades que dependem do estado? Poderia ocorrer que duas partículas estivessem no mesmo estado e, assim, tivessem também todas as propriedades dinâmicas em comum?

Podemos entender melhor essa situação comparando com o que ocorre na mecânica clássica. Neste último caso, não é possível que duas partículas tenham as mesmas propriedades dinâmicas. Elas sempre estarão em estado distintos e, além disso, podemos sempre seguir as suas trajetórias e, em diferentes momentos de tempo, identificar a mesma partícula através de sua trajetória. $O$ grande diferencial da mecânica quântica, aquele que contribuiu para promover a ideia de uma ontologia de não indivíduos, é o fato de que, além de não termos mais trajetórias bem determinadas, a representação de sistemas de múltiplas partículas é tal que, muitas vezes, não podemos atribuir um estado único para cada partícula individualmente (ou, pelo menos, não podemos fazê-lo de modo que cada partícula esteja em um estado distinto de outras, em determinadas situações). Por exemplo, dois fótons podem partilhar a relação de terem sempre a mesma polarização, quando é medida em uma dada direção, mas não se pode afirmar que um desses fótons em particular esteja polarizado.

De maneira mais abstrata, vamos considerar um caso simples de duas partículas, que vamos rotular 1 e 2 (note-se que nosso modo usual de trabalhar inicia individualizando as partículas por tais rótulos). Os possíveis estados de cada uma dessas partículas são vetores em espaços de Hilbert $\mathrm{H}_{1} \mathrm{e} \mathrm{H}_{2}$, respectivamente. $\mathrm{O}$ sistema conjunto é representado por vetores no produto tensorial $\mathrm{H}_{1} \otimes \mathrm{H}_{2}$. Se $\left\{\mathrm{v}_{\mathrm{i}}\right\}$ e $\left\{\mathrm{u}_{\mathrm{j}}\right\}$ são bases ortonormais de $\mathrm{H}_{1}$ e $\mathrm{H}_{2}$ respectivamente, então os vetores $\left\{v_{\mathrm{i}} \otimes \mathrm{u}_{\mathrm{j}}\right\}$ constituem uma base ortonormal para o produto tensorial em questão (relativamente a um adequado produto interno). Para duas partículas indiscerníveis, ou seja, duas partículas do mesmo tipo, tomamos $\mathrm{H}_{1}=\mathrm{H}_{2}$. A situação que nos interessa considerar então é se sempre podemos, em sentido análogo ao que ocorre na mecânica clássica, atribuir um estado distinto para 1 e 2. Na notação dos espaços de Hilbert que estamos considerando, devemos poder atribuir então, para a partícula 1, por exemplo, um vetor $\mathrm{v}_{1} \mathrm{em} \mathrm{H}_{1}$, e para 2, um vetor $\mathrm{u}_{2} \mathrm{em} \mathrm{H}_{2}$, de modo que o estado do sistema composto pelas duas partículas seria $\mathrm{v}_{1} \otimes \mathrm{u}_{2}$. Ainda considerando a analogia com o caso clássico, esse estado seria distinto de $u_{2} \otimes v_{1}$, pois o produto tensorial não é comutativo, ou seja, se trocarmos as partículas, então o estado do sistema composto deveria ele também mudar, o que nem sempre acontece. Qual é o problema com essa solução? 
De modo geral, a dificuldade com os vetores $v_{1} \otimes u_{2}$ e $u_{2} \otimes v_{1}$ é que eles simplesmente não representam estados possíveis para sistemas quânticos (com uma ressalva importante que será considerada abaixo). Michael Redhead chamou essas situações, oriundas do formalismo e que não têm contraparte empírica, de "estruturas excedentes" (surplus structures) (cf. French \& Krause, 2006). Deixando-se de lado o caso de para-partículas, que são possibilidades teóricas (ou seja, cuja existência é compatível com o modelo matemático adotado) sem evidência experimental atualmente, os dois únicos tipos de partículas encontrados na natureza são bósons ou férmions, respectivamente partículas com spin inteiro ou semi-inteiro. Bósons podem partilhar o mesmo estado quântico, ou seja, possuírem as mesmas propriedades físicas (sendo, portanto, nessas circunstâncias, indiscerníveis pela teoria), enquanto os férmions não podem partilhar o mesmo estado. Os vetores mencionados anteriormente, capazes de distinguir as partículas, não se prestam para representar os estados de qualquer desses dois tipos de partículas, porque não são simétricos perante uma permutação, o que deveria ocorrer pelo menos no caso de bósons que não podem ser individualizados. Para bósons, os estados possíveis são descritos por vetores da forma $1 / \sqrt{ } 2\left(v_{1} \otimes u_{2}+v_{2} \otimes u_{1}\right)$, que são simétricos relativamente a uma permutação dos índices das partículas (uma superposição de u e v). Para férmions, o único estado disponível é $1 / \sqrt{ } 2\left(v_{1} \otimes u_{2}-v_{2} \otimes u_{1}\right)$. Esse vetor é antissimétrico, trocando de sinal no caso de uma permutação, mas isso não importa, pois, em ambos os casos, o que interessa é o quadrado do módulo desses vetores, que fornece as probabilidades relevantes de acordo com a famosa regra de Born. Assim, para o cômputo das probabilidades, qualquer permutação é vista como não conduzindo a previsões distintas; como diziam os precursores da teoria, como Heisenberg e Schrödinger dentre outros, as entidades quânticas "perderam a individualidade" (cf. French \& Krause, 2006).

Insistindo um pouco mais nesse importante ponto, o formalismo quântico faz uso essencial de um princípio conhecido como postulado da indistinguibilidade. Segundo esse postulado, grosso modo, ao permutarmos os rótulos das partículas, não há como distinguir entre os estados iniciais, não permutados, dos estados finais, após a permutação, através de uma observação. Se representarmos os observáveis $\mathbf{O}$ por operadores hermitianos $\mathrm{O}$, e $\mathrm{P}$, que denotam os operadores de permutação de rótulos nos estados, então podemos expressar o postulado mais formalmente do seguinte modo: para qualquer estado |sñ e observável $\mathrm{O}$,

$$
\langle s|\mathrm{O}| \mathrm{s}\rangle=\langle\mathrm{Ps}|\mathrm{O}| \mathrm{Ps}\rangle=\left\langle\mathrm{s}\left|\mathrm{P}^{-1} \mathrm{OP}\right| \mathrm{s}\right\rangle \text {. }
$$

Ou seja, ao permutarmos os rótulos, os valores esperados (este é um conceito estatístico) da medida de um observável O para um sistema em um dado estado |sñ são os mesmos para o estado antes e depois da permutação P. 
Muito já se escreveu sobre esse postulado e sobre sua relação com as chamadas estatísticas quânticas (cf. French \& Krause 2006, cap. 4; French \& Rickles, 2003). No entanto, o que mais nos interessa aqui é o modo com pode ser entendido. Em uma primeira leitura, o postulado garante que os rótulos não possuem qualquer efeito, e que os estados obtidos devem ser tais que, antes e depois das permutações, os valores esperados para as medidas das grandezas físicas devem ser os mesmos. Isso foi visto por muitos como implicando que as partículas, na teoria quântica, tinham perdido sua identidade, como dito acima, ou seja, não poderiam mais ser consideradas como indivíduos (cf. French \& Krause, 2006, cap. 3). Tão comum era a adoção desse modo de ver as partículas quânticas como não indivíduos, que a posição foi batizada de "concepção recebida" acerca da não individualidade das partículas (cf. French, 1998). Esse comportamento está em clara oposição ao que ocorre no caso da mecânica clássica, na qual, mesmo partilhando todas as propriedades intrínsecas em comum, uma permutação de duas partículas dará origem a um estado distinto do original (o que acarreta a visão metafísica de que elas seriam "indivíduos", pois podem ser re-identificadas em situações distintas.

Mas, como podemos entender esta não individualidade mais rigorosamente? O primeiro ponto a ser notado é que a noção de objetos particulares sem individualidade, poderá, nesse caso, figurar como uma nova categoria ontológica, ou poderá ser uma subcategoria da categoria de objetos, desde que a categoria de objetos não esteja comprometida com a noção de critérios de identidade. Essas restrições à identidade devem ser feitas nesse caso, pois se acreditarmos que não há nada, nenhuma questão de fato que nos permita identificar uma partícula, nomeá-la ou numerá-la, todos esses artifícios que funcionam para indivíduos deixam de fazer sentido para as partículas quânticas. Assim, as partículas violam de alguma forma as principais propriedades da relação de identidade, e a lógica que se utiliza para tratar delas deve, igualmente, fazer restrições à identidade ("identidade", aqui, é tomada como sendo descrita pela lógica elementar clássica).

Essas, no entanto, são apenas as linhas gerais daquilo que se poderia chamar de uma metafísica (ou perspectiva) da não individualidade (cf. Krause, 2010; Arenhart \& Krause, 2012), onde definições mais precisas de "indivíduo" e de "não indivíduo" são articuladas. Mais estudos devem ainda ser realizados para investigar-se precisamente a relação entre a impermutabilidade e a ausência de individualidade, e ainda, para desenvolver-se uma ontologia envolvendo não indivíduos e sua relação com os indivíduos, se houver alguma. Não entraremos aqui nos detalhes dos sistemas de lógica já desenvolvidos com o propósito de acomodar não indivíduos, uma vez que já tratamos extensamente do assunto nos textos citados, mas limitar-nos-emos a mencionar que existem diversos sistemas para tais propósitos, tanto de lógica de ordem superior, como as cha- 
madas lógicas de Schrödinger, quanto de teoria de conjuntos, a chamada teoria de quase conjuntos (cf. French \& Krause, 2006 cap. 7, 8). Além disso, uma versão não reflexiva da mecânica quântica foi sugerida, tendo-se por base essas lógicas (cf. da Costa et al., 2012).

No entanto, a concepção recebida não é a única opção de perspectiva ontológica para a mecânica quântica. Recentemente, tem-se argumentado que as partículas, apesar do postulado da indistinguibilidade e da impermutabilidade, podem sim ser consideradas como indivíduos (cf. French \& Krause, 2006, cap. 4). Tal posição pode ser defendida do seguinte modo: consideremos novamente o caso das partículas 1 e 2 acima, e os estados $v_{1} \ddot{A} u_{2}$ e $v_{2} \ddot{A} u_{1}$, que não respeitam o tipo adequado de simetria, ou seja, não são nem simétricas nem antissimétricas. Como dissemos anteriormente, esses estados poderiam distinguir as partículas, mas os únicos tipos de partículas observadas até hoje na natureza, os bósons e férmions, de acordo com o formalismo, não ocorrem nesses estados. $O$ postulado de indistinguibilidade, no entanto, não proíbe a existência desse tipo de estados: eles simplesmente não ocorrem. Então, não poderíamos considerar esse postulado como apenas restringindo o acesso das partículas a esses estados? Ou seja, poderia ocorrer que, pelo postulado da indistinguibilidade, os observáveis devem sempre comutar com os operadores de permutação, e para que isso ocorra, os estados devem ser simétricos ou antissimétricos, sem que, no entanto, os estados não simetrizados sejam proibidos, pois eles simplesmente seriam inacessíveis às partículas. Assim, as partículas poderiam ser distinguidas, pelo menos em princípio, se estivessem nesses estados, mesmo que nunca estejam.

Esse tipo de argumento baseia-se na estrutura dos espaços de Hilbert utilizados para representar os sistemas físicos estudados (saliente-se que não estamos considerando aqui a mecânica quântica tal como formulada por David Bohm, que tem uma metafísica subjacente compatível com a visão de que as partículas seriam indivíduos. ${ }^{2}$ Uma característica desses espaços, a existência de estados não simetrizados, é utilizada para que se fundamente a outra perspectiva, a saber, a da possibilidade de uma ontologia de indivíduos. O primeiro desafio para essa posição é explicar o modo como podemos fundamentar a individualidade das partículas. Devemos notar que, ao obedecer ao postulado de indistinguibilidade, elas devem de certa forma ser indistinguíveis, não podendo ser distinguidas por nenhum observável. Ou seja, mesmo que possam ser a princípio individualizadas (por exemplo, ocupando diferentes posições espaço-temporais), essa individualidade deve estar fundamentada em algo distinto das propriedades dos subsistemas compondo o sistema em questão (mesmo "separadas", uma permutação não conduz a valores esperados distintos, como seria de esperar no que concerne a indivíduos).

2 Pretendemos tratar desse caso específico em outro trabalho, mas, por ora, cf. French \& Krause, 2006, p.191 ss. 
Quais são as opções para uma ontologia de indivíduos nesse caso? Aparentemente, devemos restringir-nos a um princípio de individuação que permita a indistinguibilidade qualitativa, concedendo a possibilidade de diferença numérica. As principais teorias acerca de particulares que possuem essas características são as teorias do substrato ou a adoção de alguma forma de essência primitiva (cf. Adams, 1979). Essas duas formas de responder ao problema da individualidade postulam que, além das propriedades qualitativas, os particulares ainda possuem seja um substrato que não é uma propriedade e garante a individualidade, como no caso da teoria dos substratos, seja uma essência individual, uma propriedade única para cada indivíduo que não conta como uma qualidade desse objeto. Em ambos os casos, dois objetos podem partilhar todas as qualidades, o que explica sua indistinguibilidade, mas seus substratos ou essências dão conta de sua diferença numérica. Notemos que se tratam de duas ontologias distintas, uma delas postulando um substrato e propriedades, com pelo menos duas categorias, e outra postulando essências individuais e propriedades, de modo que, a princípio, as essências poderiam ser propriedades, mas de algum tipo especial (cf. French \& Krause, 2006, cap. 4).

Destacamos essas duas possibilidades, apenas a título de exemplos, mas não as discutiremos em detalhes. $O$ ponto crucial é que a mecânica quântica não relativista é compatível com (pelo menos) essas duas metafísicas, ou ontologias, e a escolha por uma ou por outra dependerá de fatores alheios à própria física, como questões de natureza filosófica, por exemplo, de ordem lógica ou epistemológica. Assim, a física (ou uma teoria qualquer) não determina uma metafísica ou uma ontologia específica, sendo compatível com (pelo menos em princípio) mais de uma delas.

O que concluímos de tudo isso? A resposta acerca de que direção devemos seguir a partir dessa constatação dependerá de que tipo de visão temos da ciência, e de como encaramos a natureza de nossas teorias. Além disso, devemos ter em mente que diferentes posições acerca da própria metafísica podem e devem conduzir a um modo diverso de abordar estes problemas. Discutiremos este ponto na próxima seção.

\section{Perspegtivismo e ontologia}

Dada a situação descrita acima, como podemos relacioná-la com a maneira geral, apresentada na seção 1, de descrevermos as teorias científicas? Que tipo de vantagem uma abordagem perspectivista das teorias científicas nos permite?

Vamos recordar o que estamos discutindo ao longo deste artigo. Ao buscarmos dar conta de um domínio do conhecimento, criamos modelos matemáticos $\mathrm{Mm}$ que tratam da porção da realidade presente nesse domínio. Por meio de estudos fundacio- 
nistas, podemos, pelo menos em princípio, axiomatizar esses modelos matemáticos e obter teorias formais axiomatizadas, pelo menos em princípio (cf. Krause et al., 2011). Por meio da axiomatização, a lógica subjacente deve ser explicitada, e podemos levar em conta alguns problemas típicos de ontologia que se relacionam diretamente com a lógica, como por exemplo, se as entidades em questão possuem ou não possuem identidade, se possuem propriedades contraditórias, se existem atributos vagos etc. Claramente, esse é o tipo de discussão que está no centro da disputa acerca da natureza metafísica das entidades quânticas. São indivíduos (possuem condições de identidade bem determinadas)? Ou são não indivíduos (sem condições de identidade)? Ou ainda, algo para além da individualidade e não individualidade?

Como vimos na seção anterior, de um ponto de vista que se mantém restrito à mecânica quântica, não há como decidir acerca das duas opções apresentadas. A metafísica fica subdeterminada pela física. Podemos ter diversas metafísicas compatíveis com a mesma física. Utilizando a terminologia empregada anteriormente, a realidade empírica Re, descrita através da mecânica quântica, não nos permite decidir entre esses dois tipos de ontologia. Não chegamos a uma descrição única da natureza das entidades da realidade $R$. Nesse caso, poderíamos desesperar e abandonar as esperanças de descrever a realidade $R$, tal como ela é em si mesma. Mas será essa uma consequência necessária da subdeterminação da metafísica? Antes de abordarmos como uma possível resposta a essa questão poderia ser dada do ponto de vista do perspectivismo, vejamos algumas das alternativas atualmente disponíveis.

Uma possível saída consiste em adotar-se uma boa dose de otimismo metafísico e sustentar que através de raciocínios típicos da metafísica poderíamos tentar quebrar a subdeterminação em favor de uma das opções, qual sejam, de tratar os objetos quânticos como indivíduos ou como não indivíduos. De fato, podemos sustentar que o problema em questão é eminentemente filosófico, e que a mecânica quântica não foi desenvolvida para tratar exatamente desse tópico. Essa é em especial a visão de Bohr e da escola de Copenhague, para a qual a mecânica quântica nada mais é do que um dispositivo matemático que nos permite computar probabilidades, nada tendo a dizer sobre a ontologia subjacente à realidade que explora. Mas, se queremos explorar a metafísica (ontologia) subjacente, o apelo parece dever ser feito para propostas metafísicas razoavelmente desenvolvidas, como opções de tratar de não indivíduos na teoria de quase conjuntos ou versões da concepção da mecânica quântica com indivíduos que levem em conta as peculiaridades do modo como as propriedades são instanciadas na teoria (cf. da Costa et al., 2012; French \& Krause, 2006, cap. 4). Um dos modos de forçar-se uma quebra de subdeterminação por meio desse tipo de abordagem seria apelando para as virtudes teóricas de uma versão particular de uma dessas duas abordagens. Por exemplo, poderíamos sustentar que uma delas é mais econômica na ontologia, mais explica- 
tiva em certo sentido, satisfaz certos requisitos estéticos desejáveis, entre outros motivos (cf. Paul, 2012).

Há muitas dúvidas de que um programa nesse sentido possa ser razoavelmente desenvolvido, e muita disputa acerca da melhor forma de conduzir-se as investigações metafísicas, principalmente acerca da plausibilidade de fazer apelo às virtudes epistêmicas no caso de teorias metafísicas, quando, de fato, isso constitui apenas uma das possibilidades de desenvolvimento desse tipo de saída para a subdeterminação. De qualquer modo, é uma linha possível, mesmo que não seja a mais popular entre os filósofos hoje em dia.

Alguns filósofos propõem um meio termo diante da subdeterminação, a saber, abandonar a discussão em termos de objetos e concentrar-se nas relações que esses objetos alegadamente mantêm. Nesse sentido, seríamos realistas acerca das relações, mas não dos objetos, daí o nome dado a essa corrente, ou seja, "realismo estrutural ontológico" (cf. Ladyman, 1998, 2009). O grosso da proposta consiste em fazer-nos notar que ambas as abordagens possuem em comum o fato de empregarem determinadas relações entre os itens com os quais a mecânica quântica alegadamente trata. Assim, podemos deixar de lado a disputa acerca da natureza desses itens e manter apenas o que há em comum entre as duas abordagens, a saber, as relações. Com isso, estaremos afirmando algo mais modesto, mas podemos aparentemente estar mais seguros de sua verdade; o que supera a subdeterminação, ao colocá-la como um problema formulado de modo equivocado, como um pedido por respostas acerca da natureza de entidades que são apenas secundárias de um ponto de vista metafísico.

Todavia, apesar do seu grande apelo inicial, o realismo estrutural ontológico possui seus próprios problemas e dificuldades. Pode-se argumentar que as relações sem os objetos relacionados são apenas uma nova criação metafísica que substitui um problema (a investigação da natureza metafísica dos objetos quânticos) por outro; afinal, como podemos caracterizar relações sem objetos relacionados de modo metafisicamente satisfatório? O que nos garante que não existirão modos alternativos de darmos definições de estruturas adequadas ao realismo estrutural opntológico que são, por sua vez, empiricamente equivalentes? Dadas essas dificuldades, apesar de ser uma alternativa plausível, não há como não vermos ainda o realismo estrutural como mais uma opção metafísica, mas apenas como mais uma perspectiva no debate que, sem a força de algum tipo de argumento metafísico semelhante àquele que espera o otimista metafísico, não pode ser dita desbancar suas rivais. Assim, o próprio realismo estrutural ontológico deveria ser visto como uma solução em busca de argumentos, do mesmo modo que as abordagens de indivíduos e de não indivíduos.

A alternativa que estamos propondo neste trabalho é uma forma de perspectivismo. Cada uma das opções mencionadas é um “olhar” diferente para o domínio quân- 
tico, revelando uma de suas facetas, e merece ser estudada por si, pois cada uma delas mostra-nos um aspecto de uma solução para o problema. Os estudos fundacionais permitem que se esclareçam as bases conceituais das teorias informais, mas acabam gerando alternativas empiricamente equivalentes com metafísicas subjacentes possivelmente incompatíveis. Antes de rejeitar alguma dessas alternativas de modo dogmático, propomos que devem ser estudadas e desenvolvidas. Isso pode ajudar-nos a chegar a resultados cada vez mais precisos, como ocorre com a formulação da mecânica quântica como uma teoria de quase conjuntos (cf. da Costa et al., 2012) ou, como ocorre com a rejeição de formas fortes do princípio da identidade dos indiscerníveis na teoria quântica, de modo que uma teoria da individualidade conhecida como teoria de feixes, quando baseada nesse princípio, torna-se inviável na teoria (cf. French \& Krause, 2006, cap. 4). Mais resultados desse tipo talvez sejam possíveis no futuro, com o desenvolvimento rigoroso das diversas perspectivas. Podemos encontrar boas razões para abandonar algumas dessas abordagens, e boas razões poderiam haver para sugerir que uma delas é melhor do que as outras.

Mas isso ainda não nos responde a pergunta crucial acerca do que podemos dizer das diversas perspectivas e sua relação com $R$ ? Podemos afirmar que cada uma delas capta algum aspecto de $R$ que as outras deixam escapar? Podemos ser realistas e perspectivistas ao mesmo tempo? De fato, podemos notar que o perspectivismo pode ser empregado por realistas e por antirrealistas. Um realista pode sugerir que diversas abordagens de um mesmo domínio sejam estudadas, até que se decida por sustentar uma delas como a que melhor capta o real. Isso pode ocorrer principalmente em investigações de ponta da física, em que teorias rivais competem. mas nenhum tipo de experimento pode ser suficiente para decidir entre elas. $\mathrm{O}$ antirrealista vai evidentemente sustentar que a existência das diferentes perspectivas é um claro sinal de que a ciência não deve ser encarada como descrevendo o real em seus aspectos não observáveis, mas apenas como empiricamente adequada (ou algo que o valha). Não queremos aqui comprometer-nos com uma das duas abordagens. O perspectivismo pode ser encarado como uma abordagem de investigação que nos permite permanecer de juízo suspenso acerca desses debates, investigando cada uma das opções e obtendo razões para endossar uma ou outra das possibilidades conforme as evidências apareçam.

Para ilustrar melhor o tipo de dilema que o perspectivismo permite entender de modo mais claro, vamos considerar outro tipo de situação, na qual diferentes perspectivas podem desempenhar um papel central, o caso já mencionado em que um mesmo domínio, que chamaremos de " $D$ ”, é estudado a partir de teorias que utilizam escalas distintas. 


\section{Perspectivismo e Reducionismo}

Conforme mencionamos na introdução, existe uma situação na qual diferentes teorias vão buscar dar conta de uma mesma realidade, tratando-a, todavia, a partir de pontos de vista diferentes. Esse tipo de situação é interessante para pormos em teste nossa apresentação do perspectivismo, além de confrontá-lo com uma tese famosa na filosofia da ciência: o reducionismo.

Podemos apresentar o problema de modo claro, seguindo uma proposta apresentada por Rohrlich (1999), segundo a qual diferentes teorias fornecem descrições de diferentes aspectos da realidade. Podemos examinar, segundo Rohrlich, o mesmo objeto a partir de diferentes níveis cognitivos, cada um deles tratando de uma escala de comprimento, de tempo e de energia. Cada uma dessas teorias utiliza aindaseus próprios métodos, conceitos e procedimentos experimentais. Assim, seguindo o exemplo dado pelo próprio Rohrlich, podemos considerar um gato como nosso domínio $D$, digamos, como um objeto a ser estudado dos pontos de vista da biologia, da química, da física, da física de partículas etc. A princípio, a menos que adotemos uma tese reducionista muito forte, não haverá razão alguma que nos force a distinguir uma dentre essas várias descrições do gato como sendo a mais adequada, ou ainda tomarmos uma dessas descrições como sendo a mais próxima da verdade. Coloquemos a situação em pratos limpos, seguindo Rohrlich.

Meu gato está vivo e bem. Eu posso descrevê-lo por seu tamanho, cor, comportamento etc. Posso olhar para ele, tocá-lo e alimentá-lo. Eu sei que esse animal está realmente ali. Mas meu amigo biólogo veio visitar-me e informou-me que meu gato é, na verdade, nada mais do que uma coleção muito complicada de diferentes tipos de células em interação mútua e que ele pode provar isso fazendome olhar em um microscópio e ainda, se eu permitir, dissecando o meu gato. Mas o bioquímico da porta seguinte tem uma opinião bem diferente. Ele afirma que meu gato é, na verdade, nada mais do que uma reunião muito complexa de componentes orgânicos, proteínas, ácidos nucléicos etc., todos em mútuas interações. Ele pode, também, provar essa afirmativa.

De fato, o físico conhece ainda mais. Um gato, como toda matéria viva ou morta, é, em última análise, realmente nada mais do que átomos ligados para formar moléculas complicadas das quais o meu amigo bioquímico está falando a respeito. E ele também pode provar isso. Mas, finalmente, não podemos ignorar as pessoas que se dedicam à física de partículas, que nos dirão que o gato é realmente, realmente, nada mais do que uma reunião muito complicada de poucos tipos de partículas elementares em mútua interação. 
Perspegtivismo na filosofia da GiÊncia...

A estória termina aqui, mas apenas temporariamente. A teoria final de Weinberg [ele se refere à teoria unificada do modelo padrão da física de partículas com a mecânica quântica, algo ainda não alcançado] pode estar na esquina e, então, saberemos o que meu gato é realmente, realmente, realmente (Rohrlich, 1999, p. 357).

Desse modo, de acordo com o nível cognitivo que adotarmos, estaremos comprometendo-nos com uma descrição da realidade que envolve gatos, células, moléculas, átomos, etc. A divisão em níveis cognitivos, sem assumir o reducionismo, permite que evitemos um problema de compromisso implícito que se apresentava para as abordagens da ontologia, que se baseiam no critério de compromisso ontológico de Quine. Se aceitamos que estamos comprometidos com a existência de um gato, como o biólogo aparentemente está, então estamos comprometidos com a existência de cada um dos componentes do gato: células, moléculas, átomos, elétrons, cordas etc. Certamente, alguns desses itens não estão implicados pela biologia considerada isoladamente e, assim, devem ser tratados por outras teorias de níveis cognitivos diferentes (são elas que descrevem propriamente o comportamento desse tipo de coisa). Cada teoria poderá assumir apenas aquilo de que necessita para mobiliar o mundo. Claro, a possibilidade fica aberta para uma defesa de alguma forma de reducionismo, supondo que alguma teoria é mais fundamental (como se sugere no caso da teoria de Weinberg), ou que até, naquilo que seria um otimismo exacerbado, seja uma teoria verdadeira final, à qual todas as outras seriam reduzidas, e que, segundo esse modo de ver, fornecer-nosia, então, a ontologia final, em ambos os sentidos do termo. Nesse caso, faria sentido manter uma posição realista extrema, sustentando que há uma única história verdadeira acerca do mundo, e que o gato em questão não passa de uma configuração em forma de gato daqueles componentes mais fundamentais da realidade.

A distinção de níveis cognitivos proposta por Rohrlich possui também um interessante efeito nesse mesmo sentido. Se considerássemos a adoção de uma abordagem naturalista da ontologia sem esse tipo de qualificação, então, aparentemente o estudo da ontologia deveria estar restrito apenas a uma teoria, a saber, aquela que nos daria pretensamente $a$ descrição última da realidade, até o momento atual. Seguindo essa abordagem, seria despropositado estudar a ontologia relativamente a diversas teorias científicas, já que muitas vezes, pelo menos no modo usual de compreendê-las, essas teorias são propostas justamente para ampliar o escopo de teorias mais antigas, ou para ultrapassar as limitações umas das outras. Nesse caso, seria razoável argumentar que as teorias mais recentes produzem melhores descrições do mobiliário último do mundo. Assim, por exemplo, não teria sentido pretender estudar a ontologia associada à mecânica clássica, já que esta foi superada pela relatividade geral em alguns domínios de aplicação. Do mesmo modo, não haveria interesse em estudar a ontologia 
da mecânica quântica ortodoxa, pois ela, aparentemente, foi superada pela teoria quântica de campos. Aqui, "superada” indica que as teorias que superaram suas antecessoras, fornecem uma descrição mais acurada dos fenômenos. Se consideramos a proposta de Rohrlich, temos então que o interesse filosófico de estudar as ontologias associadas a diferentes teorias é mantido, e uma das principais motivações para sustentar que esse tipo de interesse é legítimo pode ser percebido, quando notamos que, mesmo em um ramo da ciência como a física, ainda se aplica a mecânica clássica, a mecânica quântica ortodoxa e a teoria quântica de campos em diversas situações, dependendo de considerações pragmáticas, mesmo sabendo que, a princípio, a teoria quântica de campos engloba essas teorias como casos limites. Assim, as visões do biólogo, do bioquímico, do físico e do físico de partículas nada mais são do que diferentes perspectivas sobre o gato de Rohrlich.

O perspectivista poderá, portanto, abordar esses diversos modos de descrever o mesmo objeto ou domínio da realidade utilizando-se de ferramentas conceituais que descrevem entidades em diferentes escalas sem comprometer-se com uma posição reducionista ou com qualquer uma delas em particular. Não precisará afirmar que o gato não é real, e que é real sua descrição como uma configuração de partículas elementares na forma de gato. As diversas abordagens são diversos modos de conceituarse a realidade $R$, e se essas conceituações atingem ou não $R$ é algo que não podemos garantir. Desse modo, o perspectivismo permite-nos uma espécie de neutralidade quanto a esses tópicos.

Aqui, assim como na seção anterior, a dificuldade é saber se uma abordagem perspectivista é pelo menos compatível com abordagens realistas e antirrealistas na filosofia da ciência. Aparentemente, o perspectivismo é muito mais afim ao antirrealismo, segundo o qual a ciência apenas nos conta como o mundo poderia ser, de forma que nada podemos afirmar acerca de como ele é em si. Todavia, o realista também pode sustentar uma abordagem perspectivista. Basta nesse caso não aderir a uma forma de reducionismo estrito, segundo a qual toda a realidade e as teorias científicas que dela dão conta podem ser reduzidas aos termos de uma teoria fundamental. Nesse caso, as diferentes perspectivas podem harmonizar-se entre si e cobrir simplesmente escalas distintas de objetos igualmente reais. Evidentemente, até que ponto esse modo de ver a descrição científica do mundo corresponde a alguma forma desejável de realismo é algo que deverá ser investigado tornando-se mais precisa a própria relação entre o realismo e o reducionismo, uma discussão que não enfrentaremos aqui.@ 
Décio Krause

Professor do Programa de Pós-Graduação em Filosofia,

Universidade Federal de Santa Catarina.

Bolsista de Produtividade em Pesquisa do Conselho Nacional

de Desenvolvimento Científico e Tecnológico, Brasil.

deciokrause@gmail.com

Jonas Rafael Begker Arenhart

Professor do Departamento de Filosofia,

Universidade Federal da Fronteira Sul, Campus Chapecó, Brasil.

jonas.becker2@gmail.com

\title{
Perspectivism in philosophy of science: a case-study in quantum physics
}

\begin{abstract}
In this article we present a particular view of the development of scientific theories which (following Ortega y Gasset), we term "perspectivism". Making use of this view, we discuss how we can accommodate distinct and apparently incompatible descriptions of a supposed reality under investigation. We distinguish between a "reality" $(\mathrm{R})$ and its "empirical description", $\left(\mathrm{R}_{\mathrm{e}}\right)$. Acknowledging that we can have diverse empirical descriptions of the same reality, we discuss the particular case in which the view is applied to current debates on the ontology of quantum physics, especially of non-relativistic quantum mechanics. As it is well known, this theory is compatible with treating quantum objects either as individuals or as non-individuals. Both ontologies are possible and deserve to be developed. We propose that the perspectivism developed here is neutral not only with respect to the choice of an ontology, but also concerning the debates among realists and anti-realists in the philosophy of science. We conclude that to go beyond the pluralism accepted by perspectivism, and to adopt realism or anti-realism, involves commitment to strong theses about the relation between reality and its descriptions.
\end{abstract}

KeYwords • Perspectivism. Quantum mechanics. Realism. Anti-realism. Quantum ontology. Quantum metaphysics.

\section{REFERÊNGIAS BIBLIOGRÁFICAS}

Adams, R. M. Primitive thisness and primitive identity. The Journal of Philosophy, 76, 1, 1979. p. 5-26.

Arenhart, J. R. B. \& Krause, D. Indistinguibilidade, não reflexividade, ontologia e física quântica. Scientiae Studia, 10, 1, p. 41-69, 2012.

Aurang, S., How is quantum field theory possible? Princeton: Princeton University Press, 1995.

Brading, K. \& Casteldani, E. (Ed.). Symmetries in physics: philosophical reflections. Cambridge: Cambridge

University Press, 2003. 
Bridges, D. Constructive mathematics. In: Zalta, N. (Ed.). The Stanford encyclopedia of philosophy, 2012. Disponivel em: 〈http://plato.stanford.edu/archives/fall2012/ entries/mathematics-constructive/>. Acesso em: 20 jun. 2012.

CAo, T. Y. (Ed.). Conceptual foundations of quantum field theory. Cambridge: Cambridge University Press, 1999.

Castellani, E. (Ed.). Interpreting bodies: classical and quantum objects in modern physics. Princeton: Princeton University Press, 1998.

da Costa, N. C. A. \& Krause, D., The logic of complementarity. In: van Benthem, J.; Heinzmann, G.; Rebuschi, M. \&Visser, H. (Ed.). The age of alternative logics: assessing philosophy oflogic and mathematics today. Dordrecht: Springer, 2006. p. 103-20.

Physics, inconsistencies, and quasi-truth, Synthese. No prelo.

Da Costa, N. C. A. et al. Sobre uma fundamentação não reflexiva da mecânica quântica. Scientiae Studia, 10,1, p. $71^{-104}, 2012$.

D'Espagnat, B. Veiled reality: an analysis of present day-quantum mechanical concepts. Colorado: Westview Press, 2003.

On physics and reality. Princeton: Princeton University Press, 2006.

Devitt, M. Underdetermination and realism. Philosophical Issues, 12, p. 26-50, 2002.

Falkenburg, B. Particle metaphysics: a critical account of subatomic reality. Berlin/ Heidelberg: SpringerVerlag, 2007.

French, S. On the withering away of physical objects. In: Castellani, E. (Ed.). Interpreting bodies: classical and quantum objects in modern physics. Princeton: Princeton University Press, 1998. p. 93-113.

Metaphysical underdetermination: why worry? Synthese, 180, p. 205-21, 2011.

French, S. \& Krause, D. Identity in physics. A historical, philosophical and formal analysis. Oxford: Oxford University Press, 2006.

French, S. \& Rickles, D. Understanding permutation symmetry. In: Brading, K. \& Castellani, E. (Ed.). Symmetries in physics: philosophical reflections. Cambridge: Cambridge University Press, 2003. p. 21238.

Ortega y Gasset, J. El espectador. Madrid: Editorial EDAF, 1998.

Groner, R.; Groner, M. \& Bischof, W. F. (Ed.). Methods of heuristics. Hillsdale: Erlbaum, 1983.

Heisenberg, W. A parte e o todo: encontros e conversas sobre física, filosofia, religião e política. Rio de Janeiro: Contraponto, 1996.

Krause, D. The metaphysics of non-individuality. In: Krause, D. \& Videira, A. A. (Ed.). Brazilian studies in philosophy and history of science. Boston: Springer, 2010. (Studies in the Philosophy of Science, 290). cap. 19, p. 257-67.

Krause, D. \& Videira, A. A. (Ed.). Brazilian studies in philosophy and history of science. Boston: Springer, 2010. (Studies in the Philosophy of Science, 290).

Krause, D. et al. Axiomatization and models of scientific theories. Foundations of Science, 16, p. 363-82, 2011.

Ladyman, J. What is structural realism? Studies in History and Philosophy of Science, 29, 3, p. 4.09-24, 1998. . Structural Realism. In: Zalta, N. (Ed.). The Stanford encyclopedia of philosophy, 2009. Disponível em: $<$ http://plato.stanford.edu/archives/sum2009/ entries/structural-realism/〉. Acesso em: 20 jun. 2012.

Mortensen, C. Inconsistent mathematics. In: ZaLta, N. (Ed.). The Stanford encyclopedia of philosophy, 2008. Disponível em: <http://plato.stanford.edu/archives/fall2,008/entries/mathematics-inconsistent/>. Acesso em: 20 jun. 2012.

PAul, L. A., Metaphysics as modeling: the handmaiden's tale. Philosophical Studies, 160, 1, p. 1-29, 2012.

Popper, K. R. Three worlds. Michigan: The Tanner Lecture on Human Values, Delivered at the University of Michigan, $197^{8}$. 
Perspectivismo na filosofia da Giência...

Rohrlich, F. On the ontology of QFT. In: CAo, T. Y. (Ed.). Conceptual foundations of quantum field theory. Cambridge: Cambridge University Press, 1999. p. 357-67.

Suppes, P. Heuristics and the axiomatic method. In: Groner, R.; Groner, M. \& Bischof, W. F. (Ed.). Methods of heuristics. Hillsdale: Erlbaum, 1983. p. 79-88.

. Representation and invariance of scientific theories. Stanford: Center for the Study of Language and Information, 2002.

van Benthem, J.; Heinzmann, G.; Rebuschi, M. \& Visser, H. (Ed.). The age of alternative logics: assessing philosophy of logic and mathematics today. Dordrecht: Springer, 2006.

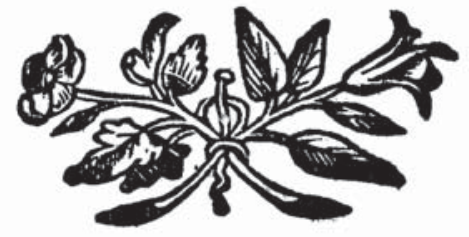

